\title{
Prostatectomía radical laparoscópica. ¿Sólo en Centros de excelencia?
}

\author{
J.I. Pascual Piédrola, V. Grasa Lanau, J.A. Cuesta Alcalá, J. Aldave Villanueva, \\ M. Ponz González, A. Ipiens Aznar
}

Servicio de Urología. Hospital de Navarra. Pamplona.

Actas Urol Esp 2005; (10): 927-933

\section{RESUME N}

PROSTATECTOMÍA RADICAL LAPAROSCÓPICA. ¿SÓLO EN CENTROS DE EXCELENCIA?

Objetivos: Presentar la experiencia adquirida en la técnica de la prostatectomía radical laparoscópica en un hospital general con un área de influencia de 200.000 habitantes, analizando los resultados preliminares de nuestra serie.

Material y Métodos: Entre diciembre de 2002 y agosto de 2005 se seleccionaron un total de 26 pacientes para prostatectomía radical laparoscópica, 25 vía transperitoneal y 1 extraperitoneal. La edad media fue de 62,3 \pm 5,3 años (rango 52-69). El PSA medio fue de 9,16 $\pm 4,2 \mathrm{ng} / \mathrm{ml}$ (rango 5,26-24,3). El estadio clínico preoperatorio fue T1c en $10(38,4 \%$ ) T2a en 8 (30,8\%) y T2b en $8(30,8 \%)$ pacientes respectivamente. La media preoperatoria del grado de Gleason fue de $6 \pm 0,8$ (rango 4-7). Tres pacientes tenían bloqueo neoadyuvante en el momento de la cirugía (11,5\%). En 2 casos se realizó linfadenectomía bilateral simultánea. No se realizó en ningún caso la técnica de preservación de nervios erectores.

Resultados: La cirugía se completó en 20 pacientes. El seguimiento medio ha sido de 12,4 $\pm 8,6$ meses (rango 3-34). No hubo muertes derivadas de la cirugía. En los primeros 10 casos hubo 6 reconversiones. En los 16 restantes 0 . El tiempo quirúrgico medio para toda la serie fue de 303,5 \pm 95,1 minutos (rango 150-540). En los 14 primeros casos el tiempo quirúrgico medio fue $332,14 \pm 92,58$ y 236,6 $\pm 66,5$ minutos en los últimos 6 casos (p<0.02). El sangrado medio fue de 90,25 $\pm 46,5$ cc (rango 40250). Complicaciones postoperatorias observamos en 4 pacientes (15,38\%). El estadio patológico fue pT2a en 3 (15\%), pT2b en $10(50 \%)$, pT3a en $3(15 \%)$ y pT3b en $4(20 \%)$ pacientes respectivamente. Márgenes positivos se encontraron en 3 casos (11,5\%): 1 pT2b (ápex), 1 pT3a y 1 pT3b. La estancia media fue de 4,7 $\pm 3,1$ días (rango 3-18). La sonda vesical se mantuvo de media 12,7 $\pm 2,7$ días (rango 7-21). Descontados los pacientes que precisaron reconversión, el 85\% (17 pacientes) referian continencia (no compresa) a los 3 meses de seguimiento.

Conclusiones: La experiencia de nuestro grupo en el manejo de otros procedimientos de cirugía laparoscópica urológica avanzada, ha permitido que la prostatectomía radical laparoscópica sea una técnica factible de realizar en un entorno de baja adscripción poblacional, consiguiendo una rápida curva de aprendizaje y con unos resultados similares a los de la cirugía convencional.

Palabras clave: Laparoscopia. Cáncer de próstata. Prostatectomía radical. Excelencia.

\section{ABSTRACT}

LAPAROSCOPIC RADICAL PROSTATECTOMY. ONLY AT CENTERS OF EXPERTISE

Objectives: To present and evaluate our experience and initial results in radical laparoscopic prostatectomy which we have been using for two and an half years in our General Hospital (covering a total population of 200.000 inhabitants).

Material and Methods. Between December 2002 and August 2005, were selected 26 patients for radical laparoscopic prostatectomy (25 transperitoneal and 1 extraperitoneal). Mean age was $62.3 \pm 5.3$ years (range 52-69). Mean PSA level was $9.16 \pm 4.20$ $\mathrm{ng} / \mathrm{mL}$ (range 5.26 to 24.3). Pre-operative classification was T1c in 10 patients (38.4\%), T2a in 8 (30.8\%) and T2b in 8. Mean Gleason Score was $6 \pm 0.8$ (range 4-7). Three patients had undergone preoperatively neo-adjuvant hormonal blockage (11.5\%). Simultaneous bilateral inguinal lymphadenectomy was performed in two patients. In no case did we consider a nerve sparing approach.

Results. The procedure was accomplished in 20 patients. Mean follow-up was $12.4 \pm 8.6$ months (range 3 - 34). There was no perioperative mortality. Conversion to open surgery was required six times in the ten first cases but not thereafter. Mean operating time for the whole series was $303 \pm 95$ minutes (range 150-540) but with a distinct difference between the first fourteen and the last six cases: $332 \pm 92,58$ versus $236.6 \pm 66.5(\mathrm{p}<0.02)$. Mean blood loss was $90.25 \pm 46.5$ ml. There were early postoperative complications in 4 patients (15.38\%). Final pathological staging was: T2a in three patients (15\%), T2b (50\%) in 10, T3a $(15 \%)$ in three and T3b (20\%) in four. Positive margins were found in three cases (11.5\%): T2b, T3a and T3b. Mean hospitalisation time was $4.7 \pm 3.1$ days. Provided there was no urinary leakage, the urinary catheter was removed at $12.7 \pm 2.7$ days. Full continence at three months was achieved in 17 of the patients $(85 \%)$ who had undergone the full laparoscopic procedure.

Conclusions: Our previous experience in other major laparoscopic procedures has allowed us to perform radical laparoscopic prostatectomy with a fast learning curve even in a setting of a relatively low population. Initial long term results seem similar to those achieved with conventional surgery.

Keywords: Laparoscopy. Prostate cancer. Radical prostatectomy. Expertise. 
L a prostatectomía radical laparoscópica (PRL) está en vías de convertirse en el nuevo estándar de la cirugía del cáncer de próstata órgano-confinado. En nuestro país, aunque con un retraso de casi 5 años con respecto a los de nuestro entorno, diversos centros han iniciado con éxito un programa de adiestramiento y puesta en marcha de la técnica de la PRL. Uno de los motivos que incide de forma negativa a la hora de plantear la incorporación de un programa de PRL en un hospital es la limitación de casos previsibles-año-cirujano sobre todo en centros con baja adscripción poblacional, donde la curva de aprendizaje puede hacerse demasiado larga para obtener un rendimiento quirúrgico adecuado. En general, se considera que la introducción de la técnica de PRL en un centro debe cumplir, entre otras premisas, el que tenga la posibilidad de realizar un mínimo de 1 cirugía a la semana y que la curva de aprendizaje se haga con un mínimo de 50 procedimientos/cirujano ${ }^{1,2}$. Presentamos en este trabajo la experiencia inicial de nuestro grupo en la técnica de la PRL, donde creemos que la curva de aprendizaje se ha reducido de forma notoria gracias a la experiencia acumulada en otras técnicas laparoscópicas, que venimos realizando de forma habitual desde 1998.

\section{MATERIAL Y MÉTODOS}

Entre diciembre de 2002 y agosto de 2005 fueron seleccionados 26 pacientes consecutivos para PRL, diagnosticados previamente de adenocarcinoma de próstata órgano-confinado. Los criterios de selección para toda la serie fueron: edad $\leq 70$ años; PSA $\leq 25 \mathrm{ng} / \mathrm{mL}$; estadio clínico preoperatorio igual o inferior a T2b (Clasificación TNM, 1997). La edad media de la serie fue de 62,3 \pm 5,3 años (rango 5269) y en todos los pacientes se incluyó consentimiento informado específico y la realización de tomografía axial computarizada (TAC) y escintigrafía ósea (GGO) previo a la cirugía, así como 2 unidades de sangre de reserva.

La indicación de PRL se hizo en base al estadio preoperatorio clínicamente localizado T1c, T2a y T2b en $10(38,4 \%), 8(30,8 \%)$ y $8(30,8 \%)$ pacientes respectivamente. El PSA medio fue de 9,16 $\pm 4,2 \mathrm{ng} / \mathrm{ml}$ (rango 5,26-24,3) y la media del índice de Gleason fue de $6 \pm 0,8$ (rango 4-7). Tres pacientes estaban en tratamiento con hormonoterapia neoadyuvante un mínimo de 3 meses antes de la cirugía. En tres casos hubo antecedentes previos de cirugía abdominal $(11,58 \%)$ : 1 Bilroth II, 1 apendicectomía y 1 herniorrafia inguinal. Previo a la cirugía se preparó a los pacientes con 4 litros de solución evacuante por vía oral la tarde anterior. Se administró profilaxis antibiótica con $2 \mathrm{~g}$ de una cefalosporina de $3^{\underline{a}}$ generación, así como 40 U.I. s.c. de heparina de bajo peso molecular como profilaxis tromboembólica, que se mantuvo hasta 2 semanas postoperatorias.

\section{PROTOCOLO QUIRÚRGICO}

Durante el tiempo que nos ha llevado completar esta modesta serie, hemos ido realizando algunas modificaciones significativas en el protocolo, con el objeto de mejorar la técnica de la PRL y reducir los tiempos quirúrgicos y de utilización de quirófano hasta promedios soportables por el servicio y por el centro.

Colocación del paciente. La posición del paciente en la mesa quirúrgica ha presentado pequeñas variaciones conforme hemos ganado en experiencia. De una posición de decúbito supino con Trendelenburg exagerado (25-30) y cinchas de sujeción para prevenir el deslizamiento del paciente de la mesa de operaciones, hemos pasado a un Trendelenburg de no más de $18^{\circ}$, con un discreto pillé, colocando en la cabecera una pieza de foam (de las utilizadas por los anestesistas para las cirugías con decúbito prono) y elevando aquella, de forma que los hombros del paciente queden encajados y no permita deslizamientos (Fig. 1). Las piernas se colocan en una discreta flexión con perneras isotérmicas autoinflables para evitar tromboembolismos. La colocación de sonda nasogástrica y canulación de una vía periférica gruesa por parte del anestesista completa la preparación del paciente.

Material quirúrgico. Para realizar el pneumoperitoneo no utilizamos aguja de Veress, accediendo siempre a través de minilaparotomía periumbilical (por donde se extraerá después la pieza embolsada). Además de una pequeña caja de cirugía menor que incluya separadores tipo Farabeuf, el material laparoscópico que utilizamos es:

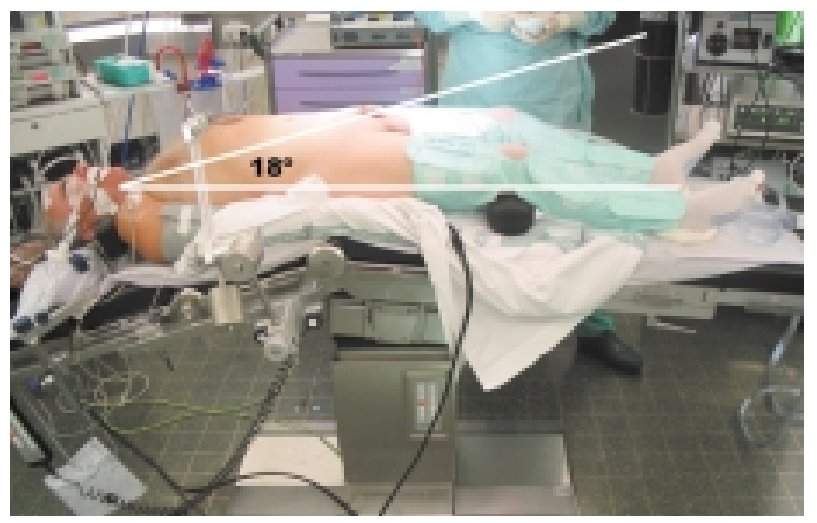

FIGURA 1. Posición en la mesa de operaciones, donde el ángulo del Trendelenburg no sobrepasa los $18^{\circ}$, evitando asi muchos de los problemas ventilatorios que pueden darse con posiciones más forzadas. 
- 3 trócares de 5 y 2 de 10

- 1 tijera laparoscópica

- 1 pinzas de coagulación bipolar

-2 pinzas atraumáticas

- 1 óptica de 0 o de $30^{\circ}$

- Brazo articulado para la óptica (Olimpus, Mod. SH-1)

- Bisturí armónico (Ultracission ${ }^{\circledR}$, Ethicon)

- Beniqué 34 Fr.

- 2 portaagujas laparoscópicos

- 1 aspirador-irrigador laparoscópico

- 1 bolsa laparoscópica

Técnica quirúrgica. En todos los casos se realizó un acceso transperitoneal, menos en uno que se hizo extraperitoneal (Prof. O. Castillo). En los primeros 19 casos realizamos la clásica técnica de Montsouris ${ }^{3,4}$ con disección de las vesículas seminales a través del fondo de saco de Douglas. En los últimos 6 casos el abordaje fue el denominado "acceso transperitoneal anterior" ${ }^{5}$ en el que una vez descolgada la vejiga, se realiza la disección de las vesículas seminales a través del cuello vesical. En dos pacientes se realizó simultáneamente una linfadenectomía ilio-obturatriz bilateral (PSA $\geq 15 \mathrm{ng} / \mathrm{mL}$ ).

La colocación de los trócares de trabajo no la hemos modificado de forma sustancial y básicamente utilizamos 2 configuraciones, dependiendo fundamentalmente de la distancia pubo-umbilical, y que vienen esquematizadas en las Figuras 2 y 3 . No hemos utilizado en ningún caso la técnica de preservación de nervios erectores y si, por el contrario, realizamos una cuidadosa disección y preservación del cuello vesical así como del ápex prostático, conforme hemos avanzado en experiencia, con el objeto de preservar al máximo la continencia urinaria. De forma resumida, los pasos que seguimos en la actualidad para la PRL transperitoneal son:

1. Sección en "U" del peritoneo parietal, que comprende lateralmente los ligamentos de Henle (arterias umbilicales obliteradas) y uraco.

2. Disección del espacio de Retzius y limpieza de la grasa periprostática.

3. Linfadenectomía ilio-obturatriz en caso de indicación.

4. Apertura de la fascia endopélvica y sección de ligamentos pubo-prostáticos.

5. Ligadura del complejo de Santorini con sutura de poliglicólico de 10.

6. Disección y preservación cuidadosa del cuello vesical.

7. Acceso al espacio retrovesical y disección de vesículas seminales.

8. Sección con bisturí armónico de pedículos prostáticos laterales.

9. Sección de la fascia de Denonvilliers y disección prerectal.

10. Sección del ápex, uretra y sección del músculo recto-uretralis.

11. Embolsado de la pieza que se abandona en fosa ilíaca hasta finalizar la intervención.

12. Sutura vésico-uretral con puntos sueltos de poliglicólico $2 / 0$ (7-8 puntos) tutorizados con beniqué o con sonda Foley.

El cirujano se coloca a la izquierda del paciente, el ayudante a la derecha y generalmente utilizamos un brazo articulado para sostener la óptica con la cámara. En el caso de un tercer ayudante, éste se coloca hacia la cabecera del paciente, en el lado derecho. La instrumentista se coloca en el lado izquierdo, próxima al cirujano. La torre de laparoscopia y los bisturís se sitúan a los pies del paciente.

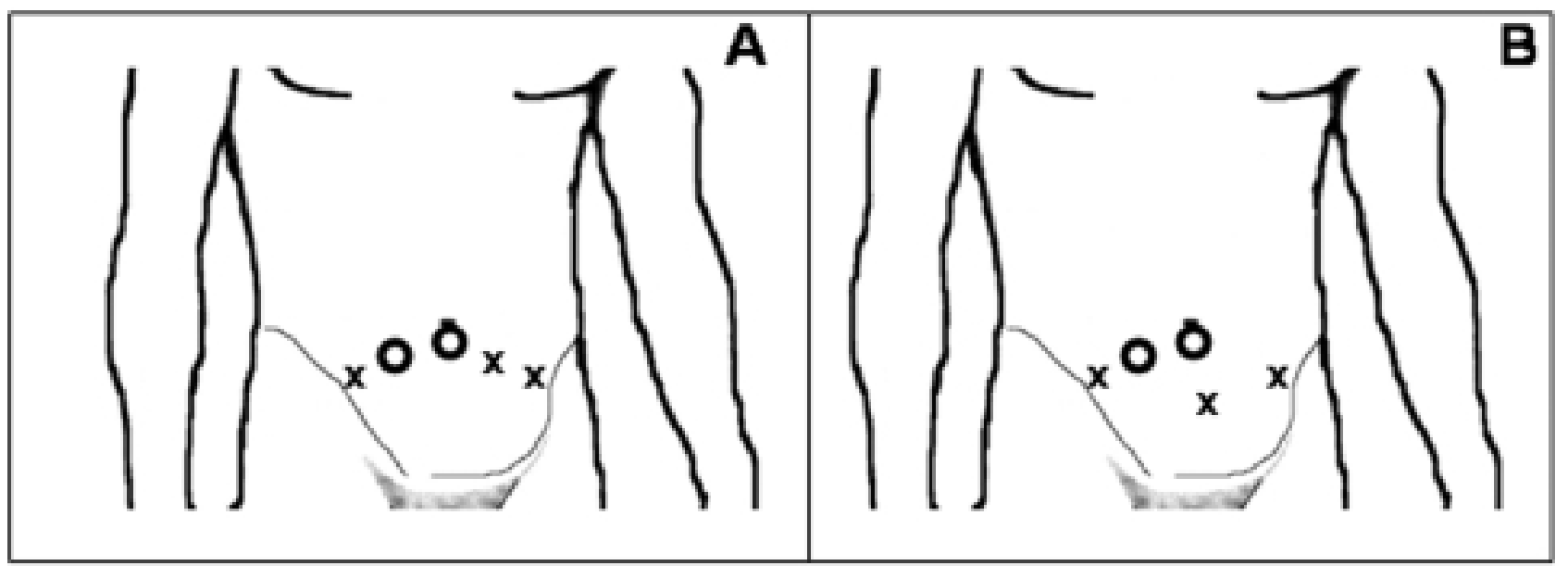

FIGURA 2. Representación esquemática de las configuraciones de los trócares en la PRL. En (A) la más habitual; en (B) la utilizada con distancias pubo-umbilicales grandes. 


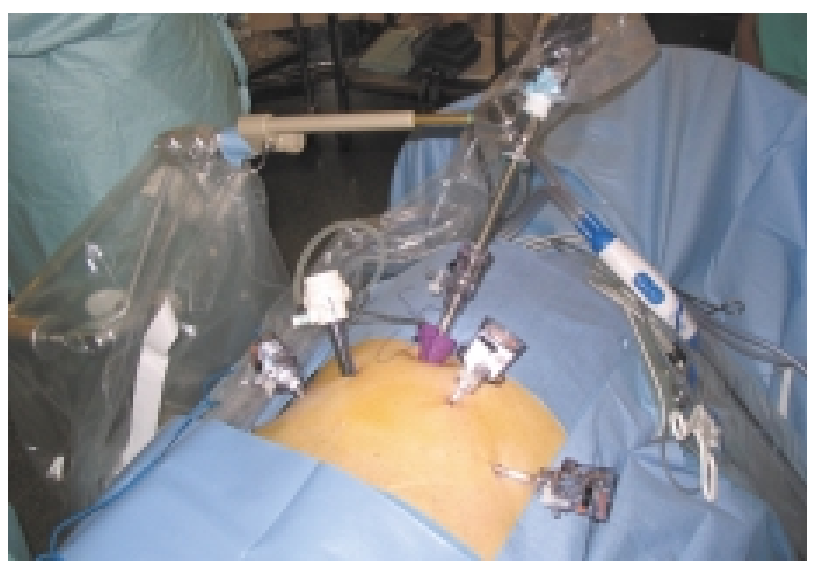

FIGURA 3. Colocación de los trócares en su configuración habitual con el brazo articulado para la óptica.

Cuidados postoperatorios. La sonda nasogástrica era retirada al finalizar la intervención quirúrgica. El drenaje se retiró en general a las 12-24 horas. La analgesia fue la necesaria para mantener un buen control del dolor y nunca se necesitaron analgésicos mayores. Se invitó a una deambulación precoz de los pacientes asî como iniciar tolerancia de líquidos a partir de las 12 horas postoperatorias. Se invitó a los pacientes a mantener medias elásticas durante la estancia hospitalaria así como en la convalecencia domiciliaria hasta 10 días postoperatorios. Se administró una dosis profiláctica diaria de heparina de bajo peso molecular (40 U.I., s.c.) que se mantuvo hasta 2 semanas postoperatorias. La retirada de la sonda vesical se realizó bajo control radiológico a partir del $7^{\circ}$ día postoperatorio.

Análisis estadístico. Los datos vienen expresados como Media \pm DE. La comparación de medias se ha realizado con el t-test de Student o el U-test de MannWhitney, considerando significativa una $\mathrm{p}<0,05$.

\section{RESULTADOS}

La PRL se pudo completar en 20 casos. En los primeros 10 casos se produjeron todas las reconversiones a cirugía abierta: dos por progresión inadecuada, un caso por I.M.C. 38, 1 caso por hipercapnia no controlada con elevación de la presión intrapulmonar, 1 perforación retrotrigonal y 1 caso con atrofia de vesícula seminal izquierda que prolongó excesivamente la disección (Tabla 1); en los siguientes 16 casos no hubo ninguna reconversión. El tiempo quirúrgico medio para toda la serie fue de 303,5 \pm 95, $1 \mathrm{~min}$. (rango 150-540). En los primeros 14 casos, el tiempo quirúrgico medio fue de 332,14 $\pm 92,58 \mathrm{~min}$. (rango 240-540), mientras que en los últimos 6 casos fue de 236,6 \pm 66,5 min. (rango 150-320, p<0,02). El sangrado promedio para toda la serie fue de 90,25 \pm 46,5 cc (rango 40-250); en los pacientes que requirieron reconversión a cirugía abierta, la media de sangrado fue de $800 \pm 209,7$ cc (rango 400-1000, p<0,0001). El peso promedio de la próstata fue de 50,69 \pm 14,53 g (rango 22-80).

$\mathrm{El}$ análisis patológico definitivo (clasificación TNM, 1997) reveló enfermedad en estadio pT2a en 3 (15\%), pT2b en 10 (50\%), pT3a en 3 (15\%) y pT3b en 4 (20\%) pacientes respectivamente (Tabla 2). En dos casos se realizó linfadenectomía ilio-obturatriz bilateral (PSA $\geq 15 \mathrm{ng} / \mathrm{ml}$ ), obteniendo un total de 8 ganglios cuyo análisis patológico fue negativo para tumor. La linfadenectomía prolongó el tiempo quirúrgico $35 \mathrm{~min}$. de promedio (30 y $40 \mathrm{~min}$. respectivamente). El índice de Gleason postoperatorio fue de media 6,4 \pm 0,85 (rango 5-8). Hubo márgenes positivos en 3 casos (11,5\%): 1 pT2b (ápex), 1 pT3a y 1 pT3b en los que se indicó RT adyuvante con bloqueo hormonal 2 años. No hubo diferencias significativas en el tiempo quirúrgico en los casos que previamente estaban con tratamiento hormonal neoadyuvante con respecto a la media.

Complicaciones intraoperatorias se produjeron en 3 casos (11,5\%) que motivaron su reconversión a cirugía abierta y que han sido descritas previamente (Tabla 1). Complicaciones postoperatorias se vieron en 4 pacientes (15,38\%): 1 íleo prolongado en un paciente con cirugía gástrica previa (Bilroth II) que motivó un ingreso de 18 días; 1 estenosis de la anas-

\section{Tabla I}

Características clínicas de 6 pacientes que requirieron reconversión

\begin{tabular}{cccccccc}
\hline Caso & Edad & PSA (ng/ ml) & Estadio clínico & $\begin{array}{c}\text { Grado } \\
\text { Gleason }\end{array}$ & $\begin{array}{c}\text { Estancia } \\
\text { (días) }\end{array}$ & $\begin{array}{c}\text { Sonda } \\
\text { (días) }\end{array}$ & Causa de reconversión \\
\hline 1 & 63 & 5.78 & T1c & 6 & 6 & 14 & Progresión inadecuada \\
2 & 67 & 8 & T1c & 6 & 6 & 14 & I.M.C..$^{(*)} 38$ \\
3 & 60 & 6.7 & T1c & 5 & 8 & 12 & Hipercapnia \\
4 & 58 & 5.59 & T2b & 6 & 8 & 14 & Atrofia v. seminal izda. \\
5 & 63 & 6.18 & T2a & 6 & 5 & 14 & Apertura vesical \\
6 & 53 & 5.5 & T1c & 6 & 5 & 12 & Progresión inadecuada \\
\hline
\end{tabular}

${ }^{(*)}$ Indice de Masa Corporal 
tomosis uretra-vesical que requirió con posterioridad una uretrotomía interna; 1 fístula rectal tardía, manifestada al 9o día como rectorragia y comprobada mediante rectoscopia, que se resolvió con medidas conservadoras (nutrición parenteral durante 12 días) y un caso de atrapamiento de la sonda vesical con un punto de sutura, resuelto de forma espontánea.

La estancia media fue de 4,7 $\pm 3,1$ días (rango 3-18). La sonda uretral se mantuvo un promedio de 12,7 \pm 2,7 días (rango 7-21) realizando en todos los casos control radiológico previo a la retirada. Un paciente falleció más de 1 año post-PRL por un carcinoma pulmonar de células grandes. El seguimiento medio de la serie fue de 12,4 \pm 8,6 meses (rango 3-34). Descontados los pacientes que requirieron reconversión a cirugía abierta, un $85 \%$ (17 pacientes) presentaban continencia (no necesidad de compresa) con un seguimiento mínimo de 3 meses. Dos pacientes precisaban 1 compresa/día y un paciente 3 .

\section{DISCUSIÓN}

En 1997 se publicó la primera serie con la técnica de la $\mathrm{PRL}^{6}$, desestimando en las conclusiones su empleo debido a la elevada morbilidad del procedimiento. Son autores franceses ${ }^{7}$ los que a partir de 1998 ponen a punto la técnica que se ha popularizado hasta hoy. En nuestra serie, en los primeros 10 casos se produjeron todas las reconversiones a cirugía abierta, unas por progresión inadecuada y otras por complicaciones intraoperatorias, datos que están en concordancia con los publicados por otros autores, que encuentran que el mayor número de reconversiones se producen en los primeros casos de la experiencia quirúrgica, sobre todo en la disección apical y la disección posterior de las vesículas seminales $^{8,9}$. El tiempo quirúrgico es un factor importante que se tiene en cuenta a la hora de plantear la incorporación de un programa de PRL en un centro. Una opción para suavizar esta distonía, que inevitablemente se produce en los servicios al comienzo de la curva de aprendizaje de la PRL por la carga quirúrgica que previamente existe, sería realizar de modo programado su implantación por fases como

Tabla 2

Características anatomopatológicas pre- y postoperatorias

\begin{tabular}{lcc}
\hline & Preoperatorio $(\mathbf{n = 2 6})$ & Postoperatorio $(\mathbf{n = 2 0})$ \\
\hline pT1c & 10 & 3 \\
pT2a & 8 & 10 \\
pT2b & 8 & 3 \\
pT3a & & 4 \\
pT3b & & \\
\hline
\end{tabular}

propugnan Llorente et al. ${ }^{10}$. En nuestro medio, gracias a que disponíamos previamente de una experiencia en cirugía laparoscópica urológica avanzada de más de $100 \operatorname{casos}^{11}$, una vez estandarizado el procedimiento quirúrgico en los primeros pacientes, conseguimos unos tiempos quirúrgicos similares a los publicados en otras series ${ }^{12-14}$. La técnica de la PRL realizada por un cirujano con experiencia constituye un factor que influye en el acortamiento del tiempo operatorio cuando se compara con el tiempo empleado por otro novel ${ }^{15}$. En nuestra serie todos los casos han sido realizados por el mismo cirujano (J.I.P.) con experiencia previa en cirugía laparoscópica avanzada, lo que nos ha permitido reducir de forma rápida los tiempos quirúrgicos.

Las complicaciones intraoperatorias y postoperatorias de la PRL no son desdeñables cuando se refieren al período comprendido en la curva de aprendizaje. Así, series ya históricas como la de Heilbronn ${ }^{16}$ comunican un 13,7\% de complicaciones intraoperatorias en sus primeros 219 pacientes, con un promedio de transfusión del $31 \%$ en los primeros 180 casos operados mediante PRL (17). En la serie de Montsouris ${ }^{4}$ se recogen un $17,1 \%$ de complicaciones mayores y menores en un total de 567 pacientes, con una tasa de transfusión del 4,9\%. La serie del grupo de Créteil publicada en $2001^{14}$ comunica en un total de 200 pacientes un promedio de complicaciones del $22,5 \%$ en los primeros 40 casos y del 3,2\% en los siguientes 94 , pero sólo una tasa de transfusiones del $4,3 \%$ para todo el grupo. Otros autores comunican complicaciones entre el 5 y $32 \%^{18-20}$. En nuestra serie, la tasa global de complicaciones fue del 26,88\%, algo más elevada que las series clásicas, con un promedio de complicaciones intraoperatorias del 11,5\%, todas al comienzo de la técnica, que motivaron la reconversión a cirugía abierta y que probablemente ahora podrían ser controladas de forma laparoscópica. El sangrado fue significativamente mayor en aquellos pacientes que precisaron reconversión, que en los que se completó la PRL en los que no fue necesario realizar transfusión de sangre. Estos datos confirman los aportados por otros autores en relación al buen control de la hemostasia que se obtiene en la PRL. Las complicaciones postoperatorias se observaron en el $15,38 \%$ de casos, cifra baja considerando las reportadas por otros autores ${ }^{19}$.

Las lesiones rectales, una de las complicaciones intraoperatorias más temidas, se describen entre el $1 \mathrm{y}$ $2,4 \%$ según las series ${ }^{21,22}$. Generalmente cuando se reconoce la lesión intraoperatoria, es factible repararla mediante sutura laparoscópica en dos planos, sin observarse incremento en la morbilidad postoperatoria. 
La localización de la lesión rectal se produce normalmente en la zona apical y por ello, el factor de mayor riesgo para la lesión parece ser la no conservación de las bandeletas que incluyen los nervios erectores ${ }^{23}$. En nuestra serie encontramos una fístula rectal tardía, probablemente producida como consecuencia de la utilización del bisturí armónico o bien por la coagulación bipolar, resuelta de forma conservadora. No hemos observado hasta el momento lesión rectal intraoperatoria a pesar de no realizar técnica de conservación de bandeletas.

De la misma forma que ocurre con la prostatectomía radical abierta, la PRL presenta márgenes quirúrgicos positivos, generalmente por un infraestadiaje que contribuye a la aparición de estos márgenes positivos. En 1998, el promedio de márgenes positivos para la prostatectomía radical abierta se situaba en torno al 28\%, con promedios del 5-36\% para estadio pT2 y 8-53\% para tumores en estadio pT3 ${ }^{24}$. En diversas series de pacientes en los que se realizó una PRL, el promedio de márgenes quirúrgicos positivos fue del 19-23\% del total, 2,1-18\% en pT2 y 30-69\% en pT3 ${ }^{25}$. En un análisis de los resultados oncológicos de las PRL realizadas en el año 2000 por el grupo de Montsouris, encuentran una disminución estadísticamente significativa en el promedio de márgenes positivos en la PRL comparada con la cirugía abierta, en particular en estadio pT2 ${ }^{26}$. En general las grandes series ya publicadas confirman que la PRL proporciona resultados satisfactorios en cuanto a control local del tumor y es competitiva en términos oncológicos con respecto a la cirugía abierta ${ }^{27,28}$. En nuestra corta serie, encontramos márgenes positivos en el $11,5 \%$ del total, $3,84 \%$ en estadio pT2 y $7,69 \%$ en estadio pT3, cifras bajas considerando las series publicadas, probablemente más en relación con la selección de los casos y la no realización de la técnica de conservación de las bandeletas, que con una excelencia quirúrgica.

La continencia en la PRL varía según las series publicadas, oscilando entre el 80 y el $95 \%$ de $\operatorname{casos}^{23}$, no encontrando diferencias significativas con la cirugía abierta $^{28}$. En nuestra serie, con un $85 \%$ de pacientes continentes un mínimo de 3 meses después de la PRL, son cifras a priori totalmente competitivas con la cirugía abierta, aunque se precisa un seguimiento mayor y más casos para poder confirmar este extremo.

La PRL, un virus Europeo con potenciales globales en palabras de Jean de la Rossette ${ }^{9}$ acaba de colonizar nuestro país, donde estamos asistiendo a una auténtica metamorfosis de la enseñanza de la cirugía urológica, cuyos resultados oncológicos son superponibles a los de la cirugía clásica, con una evidente mejora para el paciente en términos de confort postoperatorio, recuperación y normalización de su vida laboral.

\section{CONCLUSIONES}

Este estudio muestra los resultados funcionales y oncológicos obtenidos en una corta serie de PRL en un centro con una baja adscripción poblacional. Los resultados, en los que se incluyen los derivados de la curva de aprendizaje, son superponibles a los obtenidos en cirugía abierta. Un factor determinante a la hora de reducir el tiempo quirúrgico es la experiencia previa del cirujano y del equipo en técnicas laparoscópicas. En nuestras manos, encontramos que la PRL es competitiva en términos funcionales y oncológicos a los obtenidos con la cirugía abierta, aunque se precisan más casos para confirmar estos hechos.

\section{REFERENCIAS}

1. Bollens R, Sandhu S, Roumeguere T, Quackels T, Schulman C. Laparoscopic radical prostatectomy: the learning curve. Curr Opin Urol 2005; 15:79-82.

2. Van Velthoven RFP. Laparoscopic radical prostatectomy: transperitoneal versus retroperitoneal approach: is there an advantage for the patient? Curr Opin Urol 2005;15:83-88.

3. Guillonneau B, Vallancien G. Laparoscopic radical prostatectomy: the Montsouris experience. J Urol 2000;163:418-422.

4. Gillonneau B, Rozet F, Cathelineau X, Lay F, Barret E, Doublet JD, et al. Perioperative complications of laparoscopic radical prostatectomy: the Montsouris 3-year experience. J Urol 2002;167:51-56.

5. Hasan WA, Gill IS. Laparoscopic radical prostatectomy: current status. BJU Int 2004;94:7-11.

6. Schuessler W, Schulam PG, Clayman RV, Kavoussi LR Laparoscopic radical prostatectomy: initial short-term experience. Urology 1997;50:854-857.

7. Gillonneau B, Vallancien G. Laparoscopic radical prostatectomy: the Montsouris technique. J Urol 2000;163:1643-1649.

8. Bhayani SB, Pavlovich CP, Strup SE, Dahl DM, Landman J, Fabrizio MD, et al. Laparoscopic radical prostatectomy: a multiinstitutional study of conversion to open surgery. Urology 2004;63:99-102.

9. De la Rosette JJMCH, Abbou CC, Rassweiler J, Laguna MP, Schulman CC. Laparoscopic radical prostatectomy: a European virus with global potentials. Arch Esp Urol 2002;55(6):603-609.

10. Llorente C, Carrera C, Sánchez M, de la Morena JM, González F, Martinez J, et al. Implantación de un programa de prostatectomía radical laparoscópica. Actas Urol Esp 2005;29(4):349-354.

11. Valdivia JG. Estado actual de la laparoscopia Urológica en España. Comunicación personal al IV Congresso Nazionale della Societá Italiana di Endourología. Sorrento, 19-21 abril,2004.

12. Jacob F, Salomón L, Hoznek A, Bellot J, Antiphon P, Chopin DK, et al. Laparoscopic radical prostatectomy: preliminary results. Eur Urol 2000;37:615-620.

13. Abbou CC, Salomon L, Hoznek A, Antiphon P, Cicco A, Saint F, et al. Laparoscopic radical prostatectomy: preliminary results. Urology 2000;55:630-634

14. Hoznek A, Salomon L, Eric L, Antiphon P, Saint F, Cicco A, et al. Laparoscopic radical prostatectomy. The Créteil Experience. Eur Urol 2001;40:38-45.

15. El-Feel A, Davis JW, Deger S, Roigas J, Wille AH, Schnorr D, et al. Laparoscopic radical prostatectomy. An analysis of factors affecting operating time. Urology 2003;62:314-318. 
16. Rassweiler J, Seemann O, Schulze M, Teber D, Hatzinger M, Frede T. Laparoscopic versus open radical prostatectomy: a comparative study at a single institution. J Urol 2003;169:16891693.

17. Rassweiler J, Sentker L, Seemann O, Hatzinger M, Rumpelt HJ. Laparoscopic radical prostatectomy with the Heilbronn technique: an analysis of the first 180 cases. J Urol 2001;166:21012108.

18. Eden CG, King D, Kooiman GG, Adams TH, Sullivan ME, Vass JA. Transperitoneal or extraperitoneal laparoscopic radical prostatectomy: does the approach matter? J Urol 2004;172:2218-2223.

19. Gregori A, Simonato A, Lissiani A, Bozzola A, Galli S, Gaboardi F. Laparoscopic radical prostatectomy: perioperative complications in an initial and consecutive series of 80 cases. Eur Urol 2003 44:190-194.

20. Amón JH, Estébanez J, Conde C, Rodriguez A, Robles A, del Valle $\mathrm{N}$, et al. Complicaciones intraoperatorias y morbilidad de la prostatectomía radical laparoscópica (PRL) durante el período de aprendizaje. Arch Esp Urol 2004;57(4):417-424.

21. Guillonneau B, Gupta R, El Fettouh H, Chatelineau X, Baumert $\mathrm{H}$, Vallancien G. Laparoscopic management of rectal injury during laparoscopic radical prostatectomy. J Urol 2003;169: 1694-1696.

22. Katz R, Borkowski T, Hoznek A, Salomon L, de la Taille A, Abbou CC. Operative management of rectal injuries during laparoscopic radical prostatectomy. Urology 2003;62:310-313.
23. Trabulsi EJ, Guillonneau B. Laparoscopic radical prostatectomy. J Urol 2005;173:1072-1079.

24. Wieder JA, Soloway MS. Incidence, etiology, location, prevention and treatment of positive surgical margins after radical prostatectomy for prostate cancer. J Urol 1998;160:299-315.

25. Salomon L, Sèbe P, de la Taille A, Vordos D, Hoznek A, Yiou R, et al. Open versus laparoscopic radical prostatectomy: Part II. BJU Int 2004;94:244-250.

26. Fromont G, Guillonneau B, Validire P, Vallancien G. Laparoscopic radical prostatectomy: preliminary pathologic evaluation. Urology 2002;60:661-665.

27. Guillonneau B, El-Fettouh H, Baumert H, Chatelineau X, Doublet JD, Fromont G, et al. Laparoscopic radical prostatectomy: oncological evaluation after 1.000 cases at Montsouris Institute. J Urol 2003;169:1261-1266.

28. Rassweiler J, Schulze M, Teber D, Seemann O, Frede T. Laparoscopic radical prostatectomy: functional and oncological outcomes. Curr Opin Urol 2004;14:75-82.

Dr. J.I. Pascual Piédrola

Servicio de Urología. Hospital de Navarra

C/ Irunlarrea 3

31008 Pamplona (Navarra)

e-mail: pascual@pulso.com

(Trabajo recibido el 5 de septiembre 2005)

\section{COMENTARIO EDITORIAL}

Este trabajo suscita en su título una pregunta de gran interés y actualidad: la conveniencia de que la prostatectomía radical laparoscópica sea realizada en centros seleccionados. Esta cuestión, aplicada a cualquier procedimiento médico, está ya abierta a debate e incluso puesta en marcha en algunos países europeos en los que se ha centralizado la realización de algunos procedimientos en centros de referencia. Esto no sólo afecta a la laparoscopia sino también a intervenciones como la cistectomía radical y la prostatectomía radical retropúbica $\mathrm{y}$, en definitiva, a cualquier procedimiento complejo e infrecuente. En nuestro medio, altamente sectorizado en áreas de salud y con barreras administrativas de difícil traspaso, la centralización de procedimientos es una cuestión que se enfrentará a importante dificultades, pero que en mi opinión, se implantará a medio o largo plazo posiblemente como consecuencia de iniciativas supranacionales.

Los urólogos, y todos los médicos, debemos irnos acostumbrando a medir objetivamente la eficacia y las complicaciones de nuestros procedimientos de tal manera que la respuesta a la pregunta de los autores probablemente venga dada por estos datos. Es decir, creo que en un futuro próximo se nos exigirá unos resultados y unas determinadas tasas de complicaciones para que un centro sea acreditado para realizar un procedimiento. Los urólogos somos muy exigentes con la prostatectomía radical laparoscópica, porque existe una alternativa abierta, pero esta exigencia debemos aplicarla a todos nuestros procedimiento diagnósticos y terapéuticos. Por ejemplo, hoy sabemos que no es necesario hacer TAC y gammagrafía ósea a todos los pacientes que van a ser operados de una prostatectomía radical, también sabemos que el tratamiento hormonal neoadyuvante no aporta ningún beneficio y no está demostrada la necesidad de administrar radioterapia adyuvante a todos los pacientes con márgenes positivos tras la cirugía.

Dicho esto y, tras una experiencia de sólo algo más 100 prostatectomías radicales laparoscópicas por vía extraperitoneal en la Fundacion Hospital Alcorcón -lo que nos sitúa aún en periodo de aprendizaje- y de no hacer ya esta cirugía por vía abierta, hay que señalar que la PRL no ha mejorado los resultados oncológicos ni funcionales de la vía abierta. En nuestra experiencia, el beneficio para el paciente radica, sólo, en un menor sangrado (transfusión en 1\%) y una estancia más corta. ¿Son estas razones suficientes para implantar un programa de PRL?. Yo creo que sí, pero volviendo al punto de discusión anterior la respuesta dependerá también de nuestra tasa de complicaciones y hay que resaltar que la PRL las tiene y pueden ser muy severas.

Por todo esto, me gustaría felicitar a los autores ya que sólo la medición atenta de la eficacia y la complicaciones nos permite saber si avanzamos en la dirección correcta en la implantación de nuevos procedimientos. 